\title{
The identities for generalized Fibonacci numbers via orthogonal projection
}

\author{
Yasemin Alp ${ }^{1}$ and E. Gökçen Koçer ${ }^{2}$ \\ ${ }^{1}$ Department of Mathematics-Computer Sciences, Faculty of Science \\ University of Necmettin Erbakan, Meram-Konya, Turkey \\ e-mail: alpalpy@hotmail.com \\ ${ }^{2}$ Department of Mathematics-Computer Sciences, Faculty of Science \\ University of Necmettin Erbakan, Meram-Konya, Turkey \\ e-mail: ekocer@erbakan.edu.tr
}

Received: 30 August $2018 \quad$ Revised: 18 January $2019 \quad$ Accepted: 25 January 2019

\begin{abstract}
In this paper, we consider the space $R(p, 1)$ of generalized Fibonacci sequences and orthogonal bases of this space. Using these orthogonal bases, we obtain the orthogonal projection onto a subspace $R(p, 1)$ of $\mathbb{R}^{n}$. By using the orthogonal projection, we obtain the identities for the generalized Fibonacci numbers.
\end{abstract}

Keywords: Fibonacci numbers, Orthogonal basis.

2010 Mathematics Subject Classification: 11B39, 15A03.

\section{Introduction}

The generalized Fibonacci numbers $G_{n}$ are defined by the following recurrence relation for $n \geq 1$

$$
G_{n+1}=p G_{n}+G_{n-1}
$$

with the initial conditions $G_{0}=a, G_{1}=b$. The characteristic equation of recurrence (1) is

$$
\lambda^{2}-p \lambda-1=0 .
$$

The Binet's formula for the generalized Fibonacci numbers $G_{n}$ is

$$
G_{n}=A \alpha^{n}+B \beta^{n},
$$


where $\alpha$ and $\beta$ are roots of the equation (2) and $A=\frac{(b-a \beta)}{\alpha-\beta}, B=\frac{a \alpha-b}{\alpha-\beta}$.

In particular, taking $a=0$ in the initial conditions of the generalized Fibonacci number, we obtain the sequence $\left\{u_{n}\right\}$. Namely, the sequence $\left\{u_{n}\right\}$ is defined as

$$
u_{n+1}=p u_{n}+u_{n-1} ; \quad u_{0}=0, \quad u_{1}=b .
$$

Similarly, taking $2 b$ and $p b$ instead of $a$ and $b$, we obtain the sequence $\left\{v_{n}\right\}$, which is defined by the following recurrence

$$
v_{n+1}=p v_{n}+v_{n-1} ; \quad v_{0}=2 b, \quad v_{1}=p b .
$$

The Binet's formula for the numbers $u_{n}$ and $v_{n}$ is

$$
u_{n}=b\left(\frac{\alpha^{n}-\beta^{n}}{\alpha-\beta}\right) ; \quad v_{n}=b\left(\alpha^{n}+\beta^{n}\right) .
$$

Also, we can give some identities between $u_{n}$ and $v_{n}$, as follows

$$
\begin{gathered}
p v_{n-2}=u_{n}-u_{n-4}, \\
u_{n} u_{n+k}=\frac{b}{p^{2}+4}\left(v_{2 n+k}+(-1)^{n+1} v_{k}\right), \\
u_{2 m}=\frac{1}{b} u_{m} v_{m}, \\
v_{n}=p u_{n}+2 u_{n-1}, \\
v_{m} v_{m+1}-u_{m} u_{m+1}\left(p^{2}+4\right)=2(-1)^{m} b^{2} p .
\end{gathered}
$$

Taking $p=k, a=0$ and $b=1$ in (1), we have the $k$-Fibonacci numbers. Also, if we take $p=2, a=b=1$ in (1), we obtain the modified Pell numbers. We can give a few values of $\left\{G_{n}\right\},\left\{u_{n}\right\}$ and $\left\{v_{n}\right\}$ sequences as the following table

\begin{tabular}{|c|c|c|c|}
\hline \hline$n$ & $G_{n}$ & $u_{n}$ & $v_{n}$ \\
\hline \hline$\vdots$ & $\vdots$ & $\vdots$ & $\vdots$ \\
\hline-3 & $b\left(p^{2}+1\right)-a p\left(p^{2}+2\right)$ & $b\left(p^{2}+1\right)$ & $-p b\left(p^{2}+3\right)$ \\
\hline-2 & $a\left(p^{2}+1\right)-p b$ & $-p b$ & $b\left(p^{2}+2\right)$ \\
\hline-1 & $b-p a$ & $b$ & $-p b$ \\
\hline 0 & $a$ & 0 & $2 b$ \\
\hline 1 & $b$ & $b$ & $p b$ \\
\hline 2 & $p b+a$ & $p b$ & $b\left(p^{2}+2\right)$ \\
\hline 3 & $b\left(p^{2}+1\right)+a p$ & $b\left(p^{2}+1\right)$ & $p b\left(p^{2}+3\right)$ \\
\hline$\vdots$ & $\vdots$ & $\vdots$ & $\vdots$ \\
\hline \hline
\end{tabular}

Now, we give the theorems about orthogonal projection. 
Theorem 1 ([2], p. 204, Theorem 5.1.4). Consider a vector $\vec{x} \in \mathbb{R}^{n}$ and subspace $V$ of $\mathbb{R}^{n}$. Then we can write $\vec{x}=\vec{x}^{\prime \prime}+\vec{x}^{\perp}$ where $\vec{x}^{\prime \prime}$ is in $V$ and $\vec{x}^{\perp}$ is perpendicular to $V$, and this represantation is unique. The vector $\vec{x}^{\prime \prime}$ is called the orthogonal projection of $\vec{x}$ onto $V$, denoted by $\operatorname{proj}_{V}(\vec{x})$. The transformation $T(\vec{x})=\operatorname{proj}_{V}(\vec{x})=\vec{x}^{\prime \prime}$ from $\mathbb{R}^{n}$ to $\mathbb{R}^{n}$ is linear.

Theorem 2 ([2], p. 206, Theorem 5.1.5). If $V$ is a subspace of $\mathbb{R}^{n}$ with orthonormal basis $u_{1}, u_{2}, \ldots, u_{m}$, then

$$
\operatorname{proj}_{V}(x)=\left\langle u_{1}, x\right\rangle u_{1}+\cdots+\left\langle u_{m}, x\right\rangle u_{m}
$$

for all $x$ in $\mathbb{R}^{n}$.

By using the equation (8), we can give the matrix of orthogonal projection as the following theorem.

Theorem 3 ([2], p. 232, Theorem 5.3.10). Consider a subspace $V$ of $\mathbb{R}^{n}$ with orthonormal basis $u_{1}, u_{2}, \ldots, u_{m}$. The matrix $P$ of the orthogonal projection onto $V$ is

$$
P=u_{1} u_{1}^{T}+\cdots+u_{m} u_{m}^{T}
$$

Theorem 4 ([4], p. 365, Theorem 6.12). The projection matrix $P$ for subspace $V$ of $\mathbb{R}^{n}$ is both idempotent and symmetric. Conversely, every $n \times n$ matrix that is both idempotent and symmetric is a projection matrix.

Many authors have investigated the second order recurrence sequences. In particular, we consider studies connected with the Generalized Fibonacci and Horadam numbers. In [6], the author defines certain sequences and gives the properties of the certain sequences. In [7], the authors consider Horadam numbers and Horadam polynomials. Also Horzum gives the properties of Horadam polynomials. In [9], the author investigates sums of Horadam sequence. In [8], the authors consider the general Fibonacci numbers and give some interesting properties. Dupree and Mathes investigate the singular values of Hankel matrices with $k$-Fibonacci and $k$-Lucas numbers. Also, they give the orthogonal projection onto the two dimensional space of $k$-Fibonacci and $k$-Lucas sequences in [3]. In [5], the authors consider the orthogonal projection onto the two dimensional space of $k$-Fibonacci and $k$-Lucas sequences in [3], and give a new proof of obtained results by Dupree and Mathes. In [1], the authors give some identities of the Pell, modified Pell and Pell-Lucas sequences via orthogonal projection.

Motivated by the above papers, we investigate the orthogonal projection onto the two dimensional space of Generalized Fibonacci sequences. We can see that the obtained orthogonal projection matrix is a Hankel matrix with the elements of $\left\{u_{n}\right\}$ sequence entries. Also, we give the identities for the Generalized Fibonacci numbers, the elements of $\left\{u_{n}\right\}$ and $\left\{v_{n}\right\}$ sequence by using the orthogonal projection matrix. Since $k$-Fibonacci and modified Pell numbers are special cases of the generalized Fibonacci number $G_{n}$, all results in this paper generalized results from $[1,3,5]$. 


\section{Main results}

Let $R(p, 1)$ denote the subspace of $\mathbb{R}^{n}$, consisting of the $\left(G_{i}\right) \in \mathbb{R}^{n}$ which

$$
G_{i+1}=p G_{i}+G_{i-1}
$$

for $i=1,2, \ldots, n$. The elements of $R(p, 1)$ whose first two coordinates $a$ and $b$ will be denoted $\left(G_{i}\right)$ and is called the Generalized Fibonacci sequence.

In this paper, we obtain the matrix of orthogonal projection onto $R(p, 1)$ as follows

$$
\frac{p}{u_{n}}\left(\begin{array}{cccc}
u_{-n+1} & u_{-n+2} & \ldots & u_{0} \\
u_{-n+2} & u_{-n+3} & \ldots & u_{1} \\
\vdots & \vdots & \ddots & \vdots \\
u_{0} & u_{1} & \ldots & u_{n-1}
\end{array}\right)
$$

for an even $n$. Let us note that, the matrix

$$
H_{u}=\left(\begin{array}{cccc}
u_{-n+1} & u_{-n+2} & \ldots & u_{0} \\
u_{-n+2} & u_{-n+3} & \ldots & u_{1} \\
\vdots & \vdots & \ddots & \vdots \\
u_{0} & u_{1} & \ldots & u_{n-1}
\end{array}\right)
$$

is called the central Hankel matrix with elements of the sequence $\left\{u_{n}\right\}$ entries. Replacing $u_{i}$ with $v_{i}$ yields $H_{v}$ the central Hankel matrix. For an odd $n$, we obtain orthogonal projection matrix onto $R(p, 1)$ which is connected with the central Hankel matrix $H_{v}$.

Theorem 5. For an even $n$, the spectral norm of $\frac{p}{u_{n}} H_{u}$ matrix is

$$
\left\|\frac{p}{u_{n}} H_{u}\right\|_{2}=1
$$

Proof. For an even $n$, the characteristic polynomial of $\frac{p}{u_{n}} H_{u}$ is

$$
\left|\lambda I-\frac{p}{u_{n}} H_{u}\right|=\lambda^{n-2}(\lambda-1)^{2} .
$$

Hence, the roots of the characteristic equation $\left|\lambda I-\frac{p}{u_{n}} H_{u}\right|=0$ are

$$
\lambda_{1,2}=1
$$

and $\lambda_{k}=0$ for $k=3,4, \ldots, n$. The spectral norm is the maximum eigenvalue of the matrix due to $H_{u}$ symetric matrix. Clearly,

$$
\left\|\frac{p}{u_{n}} H_{u}\right\|_{2}=1 .
$$


Theorem 6. For odd $n(n=2 m+1)$, the eigenvalues of the Hankel matrix $H_{v}$ are

$$
\lambda_{1}=\frac{v_{m} v_{m+1}}{p b}, \lambda_{2}=\frac{\left(p^{2}+4\right) u_{m} u_{m+1}}{p b}
$$

and $\lambda_{k}=0$ for $k=3,4, \ldots, n$. Eigenvectors of correponding eigenvalues $\lambda_{1}$ and $\lambda_{2}$

$$
u=\left(u_{-m}, \ldots, u_{0}, \ldots, u_{m}\right)^{T} \text { and } v=\left(v_{-m}, \ldots, v_{0}, \ldots, v_{m}\right)^{T} .
$$

Proof. For odd $n(n=2 m+1)$, the characteristic polynomial of $H_{v}$ is

$$
\left|\lambda I-H_{v}\right|=\lambda^{n-2}\left(\lambda^{2}-\left(\frac{2 v_{2 m+1}}{p}\right) \lambda+\frac{\left(p^{2}+4\right)\left(u_{2 m+1}^{2}-b^{2}\right)}{p^{2}}\right)
$$

The roots of the characteristic equation are

$$
\lambda_{1}=\frac{v_{m} v_{m+1}}{p b}, \lambda_{2}=\frac{\left(p^{2}+4\right) u_{m} u_{m+1}}{p b}
$$

and $\lambda_{k}=0$ for $k=3,4, \ldots, n$. The eigenvectors corresponding eigenvalues $\lambda_{1}$ and $\lambda_{2}$ are

$$
u=\left(u_{-m}, \ldots, u_{0}, \ldots, u_{m}\right)^{T}
$$

and

$$
v=\left(v_{-m}, \ldots, v_{0}, \ldots, v_{m}\right)^{T} .
$$

Theorem 7. For odd $n(n=2 m+1)$, the spectral norm of $H_{v}$ matrix is

$$
\left\|H_{v}\right\|_{2}=\left\{\begin{array}{ll}
\frac{v_{m} v_{m+1}}{p b}, & \text { mis even } \\
\frac{\left(p^{2}+4\right) u_{m} u_{m+1}}{p b}, & \text { mis odd }
\end{array} .\right.
$$

Proof. $H_{v}$ is a symmetric matrix, then the spectral norm of $H_{v}$ matrix is the maximum eigenvalue of the matrix. Thus, the spectral norm of $H_{v}$ matrix is

$$
\left\|H_{v}\right\|_{2}=\left\{\begin{array}{ll}
\frac{v_{m} v_{m+1}}{p b}, & \text { mis even } \\
\frac{\left(p^{2}+4\right) u_{m} u_{m+1}}{p b}, & \text { mis odd }
\end{array} .\right.
$$

Now, we will prove the orthogonal projection matrix of the matrix $\frac{p}{u_{n}} H_{u}$ in (9). Therefore, we consider the matrix of order 2 as follows

$$
E=\left(\begin{array}{cc}
0 & b \\
b & p b
\end{array}\right)
$$


It follows that

$$
E^{m}=b^{m-1}\left(\begin{array}{cc}
u_{m-1} & u_{m} \\
u_{m} & u_{m+1}
\end{array}\right)
$$

for all integers $m$.

Lemma 8. For any integer $t$ and any nonnegative integer $s$, we have

$$
\sum_{i=0}^{s} \frac{E^{t+4 i}}{b^{t+4 i-1}}=\frac{u_{2(s+1)}}{p b} \frac{E^{t+2 s}}{b^{t+2 s-1}} .
$$

Proof. It suffices to prove the identity

$$
\sum_{i=0}^{s} u_{t+4 i}=\frac{u_{2(s+1)}}{p b} u_{t+2 s} .
$$

Using the Binet's formula for the $\left\{u_{n}\right\}$ sequence, we have

$$
\begin{aligned}
\sum_{i=0}^{s} u_{t+4 i} & =\sum_{i=0}^{s} b\left(\frac{\alpha^{t+4 i}-\beta^{t+4 i}}{\alpha-\beta}\right) \\
& =b \frac{\alpha^{t}}{\alpha-\beta} \sum_{i=0}^{s} \alpha^{4 i}-b \frac{\beta^{t}}{\alpha-\beta} \sum_{i=0}^{s} \beta^{4 i} \\
& =b\left[\frac{\left(\alpha^{4 s+t}-\beta^{4 s+t}\right)-\left(\alpha^{4 s+t+4}-\beta^{4 s+t+4}\right)-\left(\alpha^{t-4}-\beta^{t-4}\right)+\left(\alpha^{t}-\beta^{t}\right)}{(\alpha-\beta)\left(\alpha^{4}-1\right)\left(\beta^{4}-1\right)}\right] \\
& =-\frac{1}{p^{4}+4 p^{2}}\left(u_{4 s+t}-u_{4 s+t+4}-u_{t-4}+u_{t}\right) .
\end{aligned}
$$

From the identity (3), we obtain

$$
\sum_{i=0}^{s} u_{t+4 i}=\frac{1}{p^{3}+4 p}\left(v_{4 s+t+2}-v_{t-2}\right) .
$$

Taking $n=2 s+2$ and $k=t-2$ in the identity (4), we have

$$
\sum_{i=0}^{s} u_{t+4 i}=\frac{u_{2(s+1)}}{p b} u_{t+2 s} .
$$

Theorem 9. For an even $n$, the matrix $\frac{p}{u_{n}} H_{u}$ is orthogonal projection matrix onto $R(p, 1)$.

Proof. The orthogonal projection matrix is both symmetric and idempotent. The matrix $\frac{p}{u_{n}} H_{u}$ is clearly symmetric. Therefore, we need to show only that $\frac{p}{u_{n}} H_{u}$ is idempotent. Namely, we will prove that

$$
H_{u}^{2}=\frac{u_{n}}{p} H_{u}
$$


We can express the matrix $H_{u}$ as

$$
H_{u}=\left(\begin{array}{ccccc}
\frac{E^{-n+2}}{b^{-n+1}} & \frac{E^{-n+4}}{b^{-n+3}} & \ldots & \frac{E^{-2}}{b^{-3}} & \frac{E^{0}}{b^{-1}} \\
\frac{E^{-n+4}}{b^{-n+3}} & \frac{E^{-n+6}}{b^{-n+5}} & \ldots & \frac{E^{0}}{b^{-1}} & \frac{E^{2}}{b} \\
\vdots & \vdots & \ddots & \vdots & \vdots \\
\frac{E^{-2}}{b^{-3}} & \frac{E^{0}}{b^{-1}} & \ldots & \frac{E^{n-6}}{b^{n-7}} & \frac{E^{n-4}}{b^{n-5}} \\
\frac{E^{0}}{b^{-1}} & \frac{E^{2}}{b} & \ldots & \frac{E^{n-4}}{b^{n-5}} & \frac{E^{n-2}}{b^{n-3}}
\end{array}\right)
$$

with the matrix $E^{m}$. Taking $n=2 m$, we have

$$
H_{u}=\left[\frac{E^{2(i+j)-2(m+1)}}{b^{2(i+j)-2(m+1)-1}}\right]_{i, j=1}^{m} .
$$

Using the Lemma 6, we obtain

$$
\begin{aligned}
H_{u}^{2} & =\left[\sum_{r=1}^{m} \frac{E^{2(i+r)-2(m+1)}}{b^{2(i+r)-2(m+1)-1}} \frac{E^{2(r+j)-2(m+1)}}{b^{2(r+j)-2(m+1)-1}}\right]_{i, j=1}^{m} \\
& =\left[\sum_{r=1}^{m} \frac{E^{2(i+j)-4 m+4(r-1)}}{b^{2(i+j)-4 m+4(r-1)-2}}\right]_{i, j=1}^{m} \\
& =\frac{u_{2 m}}{p}\left[\frac{E^{2(i+j)-4 m+2(m-1)}}{b^{2(i+j)-4 m+2(m-1)-1}}\right]_{i, j=1}^{m} \\
& =\frac{u_{n}}{p}\left[\frac{E^{2(i+j)-2(m+1)}}{b^{2(i+j)-2(m+1)-1}}\right]_{i, j=1}^{m} \\
& =\frac{u_{n}}{p} H_{u} .
\end{aligned}
$$

Using the idempotency of the matrix $\frac{p}{u_{n}} H_{u}$, we obtain the identity for the elements of $\left\{u_{n}\right\}$ sequence as

$$
\frac{p}{u_{n}} u_{i+j-n+1}=\frac{p^{2}}{u_{n}^{2}} \sum_{k=0}^{n-1} u_{i+k-n+1} u_{j+k-n+1}
$$

for all even $n$ and $-n+1 \leq i, j \leq n-1$. Thus, we give the following corollary.

Corollary 10. For an even $n$ and $-n+1 \leq i, j \leq n-1$, we have

$$
u_{n} u_{i+j-n+1}=p \sum_{m=0}^{n-1} u_{i-m} u_{j-m} .
$$

Now, we give the matrix of orthogonal projection onto $R(p, 1)$ for the even values of $n$. Hence, we consider the different orthogonal bases of the space $R(p, 1)$.

Assume that $n$ is even and $s=\left(G_{0}, G_{1}, \ldots, G_{n-2}, G_{n-1}\right) \in R(p, 1)$, which is a column vector. We define

$$
s^{\perp}=\left(-G_{n-1}, G_{n-2}, \ldots,-G_{1}, G_{0}\right)^{T} .
$$


It is clear that $\left\{s, s^{\perp}\right\}$ is an orthogonal basis for the space $R(p, 1)$. Normalizing $s$ and $s^{\perp}$, we consider the $t$ and $w$ vectors as

$$
t=\frac{s}{\|s\|}, w=\frac{s^{\perp}}{\left\|s^{\perp}\right\|}
$$

where

$$
\|s\|^{2}=\left\|s^{\perp}\right\|^{2}=\frac{G_{n} G_{n-1}+\left(p a^{2}-a b\right)}{p} .
$$

From Theorem 3, the matrix of orthogonal projection onto $R(p, 1)$ is

$$
P=t t^{T}+w w^{T}
$$

which is the Hankel matrix in (9).

Theorem 11. For an even $n$ and $0 \leq i, j \leq n-1$, we have

$$
u_{i+j-n+1}\left(G_{n} G_{n-1}+\left(p a^{2}-a b\right)\right)=u_{n}\left(G_{i} G_{j}+(-1)^{i+j} G_{n-i-1} G_{n-j-1}\right) .
$$

Proof. By equalizing the $i j$-th entries of the matrices in (9) and (12), we have

$$
\frac{p}{G_{n} G_{n-1}-G_{0} G_{-1}}\left(G_{i} G_{j}+(-1)^{i+j} G_{n-i-1} G_{n-j-1}\right)=\frac{p}{u_{n}} u_{i+j-n+1} .
$$

From $G_{0}=a$ and $G_{-1}=b-p a$, we obtain

$$
u_{i+j-n+1}\left(G_{n} G_{n-1}+\left(p a^{2}-a b\right)\right)=u_{n}\left(G_{i} G_{j}+(-1)^{i+j} G_{n-i-1} G_{n-j-1}\right) .
$$

By using the above theorem, we have the identities for the elements of $\left\{G_{n}\right\}$ and $\left\{u_{n}\right\}$ sequences as follows.

For $i=j=0$ and $i=j=n-1$ in (13), we obtain

$$
\frac{u_{n}}{u_{-n+1}}=\frac{G_{n} G_{n-1}+\left(p a^{2}-a b\right)}{a^{2}+G_{n-1}^{2}} .
$$

Taking $i=j=\frac{n}{2}$ in (13), we have

$$
\frac{\left(G_{n} G_{n-1}+\left(p a^{2}-a b\right)\right) b}{u_{n}}=G_{\frac{n}{2}}^{2}+G_{\frac{n}{2}-1}^{2} .
$$

Let $i=n-1, j=1$ in (13), then

$$
b\left(G_{n} G_{n-1}+\left(p a^{2}-a b\right)\right)=u_{n}\left(b G_{n-1}+a G_{n-2}\right) .
$$


Let $\alpha$ and $\beta$ be roots of the characteristic equation (2). Now, we consider the another orthogonal basis of $R(p, 1)$, which is $\{s, t\}$, where

$$
s=\left(1, \alpha, \alpha^{2}, \ldots, \alpha^{n-1}\right)^{T} \text { and } t=\left(1, \beta, \beta^{2}, \ldots, \beta^{n-1}\right)^{T}
$$

The orthogonal projection matrix onto $R(p, 1)$ is

$$
P=\frac{1}{\|s\|^{2}} s s^{T}+\frac{1}{\|t\|^{2}} t t^{T} .
$$

This matrix is a Hankel matrix with the elements of the sequence $\left\{u_{n}\right\}$ in (9).

Theorem 12. For $n$ is even, we have

$$
\frac{\alpha^{i+j+1}}{\alpha^{2 n}-1}+\frac{\beta^{i+j+1}}{\beta^{2 n}-1}=\frac{1}{u_{n}} u_{i+j-n+1} .
$$

for all $0 \leq i, j \leq n-1$.

Proof. By equalizing the $i j$-th entries of the matrices in (9) and (14), we have

$$
\frac{\alpha^{2}-1}{\alpha^{2 n}-1} \alpha^{i+j}+\frac{\beta^{2}-1}{\beta^{2 n}-1} \beta^{i+j}=\frac{p}{u_{n}} u_{i+j-n+1} .
$$

For $\alpha^{2}-1=p \alpha$ and $\beta^{2}-1=p \beta$, the result is clear.

In particular, taking $i=1$ and $j=n-1$ in (15), we have

$$
\frac{\alpha^{n+1}}{\alpha^{2 n}-1}+\frac{\beta^{n+1}}{\beta^{2 n}-1}=\frac{1}{u_{n}} u_{1} \text {. }
$$

Using $\alpha \beta=-1$ and an even $n$,

$$
\left(\frac{\alpha^{n+1}}{\alpha^{2 n}-1}+\frac{\beta^{n+1}}{\beta^{2 n}-1}\right)\left(\alpha^{n}-\beta^{n}\right)=(\alpha-\beta) .
$$

Hence, the Binet's formula for the elements of $\left\{u_{n}\right\}$ sequence appears as follows

$$
u_{n}=b\left(\frac{\alpha^{n}-\beta^{n}}{\alpha-\beta}\right) \text {. }
$$

Now, we give the matrix of orthogonal projection onto $R(p, 1)$ for the odd values of $n$, $n=2 m+1$. Therefore, we consider the eigenvectors $u$ and $v$ of the matrix $H_{v}$, which are

$$
u=\left(u_{-m}, \ldots, u_{0}, \ldots, u_{m}\right)^{T} \text { and } v=\left(v_{-m}, \ldots, v_{0}, \ldots, v_{m}\right)^{T},
$$

respectively. We have the norms of $u$ and $v$

$$
\|u\|^{2}=\frac{2 u_{m} u_{m+1}}{p} \text { and }\|v\|^{2}=\frac{2 v_{m} v_{m+1}}{p} .
$$


Also, using the fact that $u_{-i}=(-1)^{i+1} u_{i}$ and $v_{-i}=(-1)^{i} v_{i}$, we have

$$
\sum_{i=-m}^{m} u_{i} v_{i}=-\sum_{i=1}^{m} u_{i} v_{i}+u_{0} v_{0}+\sum_{i=1}^{m} u_{i} v_{i}=0
$$

Namely $\{u, v\}$ is an orthogonal basis of $R(p, 1)$. The orthogonal projection matrix onto $R(p, 1)$ is given by

$$
P=\frac{p}{2 u_{m} u_{m+1}} u u^{T}+\frac{p}{2 v_{m} v_{m+1}} v v^{T}
$$

The following theorem gives the second expression for this projection.

Theorem 13. The orthogonal projection matrix in (16) is

$$
\frac{p^{2}(-1)^{m+1}}{\left(p^{2}+4\right) u_{2 m} u_{2 m+2}} v v^{T}+\frac{p b}{u_{m} u_{m+1}\left(p^{2}+4\right)} H_{v} .
$$

Proof. Let us consider the Hankel matrix with elements of $\left\{v_{n}\right\}$ sequence entries, $H_{v}$. The eigenvalues of $H_{v}$ are $\frac{u_{m} u_{m+1}\left(p^{2}+4\right)}{p b}$ and $\frac{v_{m} v_{m+1}}{p b}$. The eigenvectors $u$ and $v$ are connected with these eigenvalues. Thus

$$
\begin{aligned}
H_{v} & =\frac{u_{m} u_{m+1}\left(p^{2}+4\right)}{p b} \frac{p}{2 u_{m} u_{m+1}} u u^{T}+\frac{v_{m} v_{m+1}}{p b} \frac{p}{2 v_{m} v_{m+1}} v v^{T} \\
& =\frac{p^{2}+4}{2 b} u u^{T}+\frac{1}{2 b} v v^{T} .
\end{aligned}
$$

The projection matrix in (16) is

$$
\begin{aligned}
P & =\frac{p}{2 u_{m} u_{m+1}} u u^{T}+\frac{p}{2 v_{m} v_{m+1}} v v^{T} \\
& =\frac{2 b}{p^{2}+4} \frac{p}{2 u_{m} u_{m+1}}\left(H_{v}-\frac{1}{2 b} v v^{T}\right)+\frac{p}{2 v_{m} v_{m+1}} v v^{T} \\
& =\left(\frac{p}{2 v_{m} v_{m+1}}-\frac{b p}{2 b\left(p^{2}+4\right) u_{m} u_{m+1}}\right) v v^{T}+\frac{p b}{\left(p^{2}+4\right) u_{m} u_{m+1}} H_{v} .
\end{aligned}
$$

Using the identities (5) and (7), we simplify the above equation. Namely, we have

$$
P=\frac{p^{2}(-1)^{m+1}}{\left(p^{2}+4\right) u_{2 m} u_{2 m+2}} v v^{T}+\frac{p b}{u_{m} u_{m+1}\left(p^{2}+4\right)} H_{v} .
$$

Corollary 14. If $n$ is odd, then

$$
\frac{p}{2 u_{m} u_{m+1}} u_{i} u_{j}+\frac{p}{2 v_{m} v_{m+1}} v_{i} v_{j}=\frac{p^{2}(-1)^{m+1}}{\left(p^{2}+4\right) u_{2 m} u_{2 m+2}} v_{i} v_{j}+\frac{p b}{u_{m} u_{m+1}\left(p^{2}+4\right)} v_{i+j}
$$

for $-m \leq i, j \leq m$. 
Proof. By equalizing the $i j$-th entries of the matrices in (16) and (17), the result is clear.

Taking $i=j=m$ in (18), we have the identity for the elements of $\left\{u_{n}\right\}$ and $\left\{v_{n}\right\}$ sequences as follows

$$
b u_{4 m+2}=v_{2 m} u_{2 m+2}-p b^{2} .
$$

\section{References}

[1] Alp, Y., \& Koçer, E. G. (2018). The Pell, Modified Pell Identities via Orthogonal Projection, Journal of Applied Mathematics and Computation (JAMC), 2 (3), 67-78.

[2] Bretscher, O. (2015). Linear Algebra with Applications, Fifth Edition, Pearson Education.

[3] Dupree, E., \& Mathes, B. (2012). Singular Values of $k$-Fibonacci and $k$-Lucas Hankel matrices, Int. J. Contemp. Math Sciences, 47 (7), 2327-2339.

[4] Fraleigh, J. B., \& Beauregard, R. A. (1995). Linear Algebra, Addison-Wesley Publishing Company.

[5] Hawkins, K., Hebert-Johnson, U., \& Mathes, B. (2015). The Fibonacci Identities of Orthogonality, Linear Algebra and Its Application, 475, 80-89.

[6] Horadam, A. F. (1965). Basic Properties of a Certain Generalized Sequence of Numbers, The Fibonacci Quarterly, 3 (3), 161-176.

[7] Horzum, T., \& Koçer, E. G. (2009). On Some Properties of Horadam Polynomials, International Mathematical Forum, 4 (25), 1243-1252.

[8] Kalman, D., \& Mena, R. (2003). The Fibonacci Numbers-Exposed, Mathematics Magazine, 76 (3), 167-181.

[9] Mansour, T. (2004). A formula for the generating functions of powers of Horadam's Sequence, Australasian Journal of Combinatorics, 30, 207-212. 